\title{
IMPLICADOS, INTERMITENTES, DESENGAJADOS? Estilos de mediação de pais de crianças de 3-8 anos que usam a internet
}

\author{
Cristina Ponte \\ ICNOVA, Faculdade de Ciências Sociais e Humanas, Universidade Nova de Lisboa, Lisboa, Portugal
}

José Alberto Simões

CICS.NOVA, Faculdade de Ciências Sociais e Humanas, Universidade Nova de Lisboa, Lisboa, Portugal

Susana Batista

CICS.NOVA, Faculdade de Ciências Sociais e Humanas, Universidade Nova de Lisboa, Lisboa, Portugal

Teresa Sofia Castro

ICNOVA, Faculdade de Ciências Sociais e Humanas, Universidade Nova de Lisboa, Lisboa, Portugal

Resumo Este artigo apresenta resultados do primeiro inquérito nacional sobre como crianças (3-8 anos) se relacionam com ecrãs em ambientes familiares, focando-se nas mediações de pais cujos filhos fazem uso da internet. Com base em literatura sobre o tema e em resultados sobre o contexto português, procedeu-se a análises estatísticas de correspondências múltiplas e de clusters que consideraram fatores do lado da criança e do lado dos pais relativamente a mediações ativas e de segurança. Da análise emergiram três tipos de mediadores - implicados, intermitentes e desengajados - cujas características se discutem e se problematizam.

Palavras-chave: crianças, famílias, mediação parental, tecnologias digitais.

Abstract This article presents results from the first national survey on how children aged 3-8 years old interact with screens in the household, focusing on mediations reported by the parents whose children use the internet. Bearing in mind the literature on the topic and the results on the Portuguese context, we conducted a statistical analysis of multiple correspondences and clusters considering factors related to children and related to parents regarding active and security mediations. Three types of mediators emerged from the analysis - involved, intermittent and disengaged - whose characteristics are discussed and problematized.

Keywords: children, families, parental mediation, digital technologies.

Résumé Cet article présente les résultats de la première enquête nationale sur les interactions des enfants âgés de 3 à 8 ans avec les écrans de leur environnement familial, en se concentrant sur les médiations rapportées par les parents dont les enfants utilisent internet. Tenant compte de la littérature sur le sujet et de résultats sur le contexte portugais, nous avons effectué des analyses statistiques de correspondances multiples et de clusters en considérant les facteurs liés aux enfants et aux parents concernant les médiations actives et de sécurité. Trois types de médiateurs ont émergé de l'analyse - impliqués, intermittents et désengagés - dont les caractéristiques sont discutées et problématisées.

Mots-clés: enfants, familles, médiation parentale, technologies digitales.

Resumen Este artículo presenta los resultados de la primera encuesta nacional sobre como los niños de 3 a 8 años interactúan con las pantallas en el hogar, centrándose en las mediaciones informadas por los padres cuyos hijos usan internet. Teniendo en cuenta la literatura sobre el tema y resultados del contexto portugués,

realizamos análises estadísticas de múltiples correspondencias y clusters que consideran los factores relacionados con los niños y los padres en relación con las mediaciones activas y de seguridad. Del análisis surgieron tres tipos de mediadores - involucrados, intermitentes y desconectados - cuyas características se discuten y problematizan.

Palabras clave: niños, familias, mediación parental, tecnologías digitales. 


\section{Introdução}

Mudam-se os tempos, muda a tecnologia, mudam as gerações de pais e de filhos. Lares com crianças estão mais apetrechados em tecnologia do que os lares em geral (INE, 2015). Aos computadores portáteis, já populares em Portugal no final da década passada (Ponte et al., 2012), vieram juntar-se tecnologias mais individualizadas, portáteis e com entrada na internet através de aplicações visualmente reconhecíveis. A forma tátil de acesso à rede torna também possível que crianças, mesmo sem saberem ler e escrever, facilmente fiquem online. Importa por isso conhecer em que condições as crianças mais novas estão a crescer entre esses ecrãs e de que modos as suas famílias intervêm (ou não) nessa orientação e acompanhamento.

Este artigo sustenta-se teoricamente na investigação sobre mediação parental relativamente aos ecrãs digitais que tem vindo a ser produzida na última década. Em particular, foi sua fonte de inspiração um recente estudo coordenado por Sonia Livingstone e Ellen Helsper que revê o modelo anterior de mediação parental, no sentido de identificar formas de mediação que, simultaneamente, não ignorem os riscos da internet que as crianças podem encontrar, e que as capacitem para que tirem partido das suas oportunidades (Livingstone et al., 2017). No modelo que os autores propõem, a uma mediação restritiva radical, que protege as crianças mas que impede também a aquisição e maturação de competências digitais, opõe-se uma mediação apresentada como capacitante, onde as crianças adquirem competências em ambientes onde o cuidado com a segurança é preservado e onde existe diálogo sobre o digital.

Este contributo teórico, complementado com a atenção ao contexto português, que apresentamos no início deste artigo, foi a base para realizarmos uma análise estatística aos resultados do primeiro inquérito nacional sobre como os pais portugueses estão a mediar os ambientes digitais experimentados por crianças mais novas. Os procedimentos metodológicos dessa análise e a descrição e discussão dos clusters encontrados ocupam a parte seguinte do artigo, a anteceder as notas finais.

\section{Estudar a mediação parental: da televisão aos dispositivos digitais}

Desde que a televisão entrou nos lares que os ecrãs são parte constitutiva de rotinas e dinâmicas de família, demarcando interações entre gerações, realizando usos e apropriações que, em última análise, as diferenciam. Os ecrãs (de maior dimensão ou os mais recentes, menores e mais individualizados) seduzem as crianças e conquistaram o espaço geográfico, social, relacional e temporal nas famílias, oferecendo possibilidades versáteis de entretenimento interativo e personalizável. De modo a encontrar um ponto de equilíbrio nesse uso dinâmico, muitos pais procuram tirar partido das vantagens educacionais e sociais, contendo efeitos nocivos de conteúdos, precavendo a segurança biopsicossocial das crianças e estimulando um desenvolvimento holístico saudável (ver Ponte et al., 2017).

Com a entrada da internet, ainda em ecrãs essencialmente fixos do computador de mesa, o acompanhamento parental ou o que se designa comonediação parental 
apoiou-se em muito do que eram estratégias e regras estabilizadas e fortemente influenciadas pela tradição televisiva.

A primeira literatura sobre mediação da internet identificava três estratégias genéricas transversais: ativa, restritiva e co-uso (Valkenburg et al., 1999; Nathanson, 1999). Estas propostas consistiam, respetivamente, na descodificação crítica de conteúdos com a criança, em práticas de controlo de modo a regrar o tempo ou os conteúdos acedidos pela criança e, simplesmente, em realizar um uso conjunto. Estudos apontavam para uma mediação parental influenciada pelo género e idade da criança, bem como o estilo parental e o estatuto socioeconómico familiar (Livingstone e Helsper, 2008). Fazendo face a evoluções tecnológicas e de apropriação, esse modelo inicial foi complementado com mais duas abordagens: a monitorização, através da verificação da atividade dos filhos na internet e a restrição técnica pelo uso de software e filtros que limitam o acesso a conteúdos inapropriados (Livingstone e Helsper, 2008).

De modo a cobrir novos contextos de ecrãs, usados de modo mais individualizado pelas crianças, a rede europeia "EU Kids Online" atualizou a classificação de estratégias de mediação parental, no inquérito realizado em 2010 (Pasquier, Simões e Kredens, 2012). Foram identificados cinco estilos de intervenção já distanciados da herança televisiva e que fundem mediação ativa e co-uso: 1) mediação ativa do uso da internet, que promove uma experiência conjunta e dialogada da internet, com foco na relação social (por exemplo, conversar sobre o que se faz); 2) mediação ativa da segurança na internet com orientações e recomendações dos pais de modo a promover um uso mais seguro e responsável; 3) mediação restritiva que assenta em limites de tempo, conteúdos e atividades online, bem como no local de uso da internet; 4) mediação técnica suportada pelo uso de software e filtros para restringir e supervisionar as atividades online; e 5) monitorização, a verificação da atividade da criança após utilização.

Os resultados desse inquérito abriram caminho para outras pesquisas, de tipo qualitativo, que procuraram ter em conta dinâmicas vividas pelas crianças (Smahel e Wright, 2014; Haddon e Vincent, 2014) e cobrir idades mais novas, captando momentos iniciais do uso de meios digitais e formas de mediação nessas idades. No trabalho empírico envolvendo 70 famílias europeias com crianças menores de 8 anos, Chaudron et al. (2015) concluíram que os estilos de parentalidade - que se apresentam frequentemente como inconsistentes (Zaman et al., 2016) - são influenciados por valores familiares, perceções, composição familiar e experiências do digital, como resultado de uso pessoal, do histórico com filhos mais velhos ou de interações entre familiares, nomeadamente entre irmãos de diferentes idades (ver também Livingstone et al., 2015).

Longe do uso como espetador de ecrã grande e fixo, colocado nos espaços comuns da casa, os média digitais móveis com ligação Wi-Fi trazem possibilidades e desafios aumentados para as famílias. Um mundo de oportunidades e riscos parece caber na mão das crianças.

Pais cujas considerações privilegiam benefícios e potencialidades das ferramentas digitais tendem a enquadrá-las no quotidiano pessoal e familiar, encorajando positivamente a sua utilização (Vittrup et al., 2016). Pais mais desassossegados 
preferem posturas alicerçadas na restrição no que toca ao tempo de ecrã, a conteúdos e ao uso de controlos técnicos (Duerager e Livingstone, 2012; Helsper et al., 2013). A mediação restritiva é a mediação elegida por famílias de estatuto socioeconómico mais baixo, com menor escolarização, digitalmente menos confiantes e que fazem menos uso da internet (Duerager e Livingstone, 2012).

A investigação tem também identificado que os diferentes estilos de mediação se articulam e que não há uma única (e correta) maneira de gerir os acessos e usos em contexto familiar. As relações pais-filhos devem refletir o reconhecimento de uma influência com caráter dialético e bidirecional. Se os pais influenciam a criança no processo de socialização para os meios digitais, a criança é, por seu lado, também um ator reconhecidamente ativo e influente da adoção e uso, em particular das novidades tecnológicas, no cenário doméstico (Nelissen e Bulck, 2017). Este processo dialógico intergeracional é designado participatory learning ou aprendizagem participativa (Clark, 2011), uma estratégia de mediação emergente em que pais e filhos interagem, experimentam e aprendem juntos com e através dos média digitais.

O reconhecimento baseado em evidência empírica de que as características e agência das crianças importam, que riscos e oportunidades digitais estão intimamente associados, e que existe uma diversidade de práticas familiares marcadas por competências digitais (de pais e de filhos), levou a uma reapreciação das cinco estratégias de mediação parental para a internet, usadas no inquérito "EU Kids Online" de 2010, procurando dar conta de articulações numa recente análise liderada por Sonia Livingstone.

Enfatizando a importância da agência das próprias crianças, Livingstone et al. (2017) exploram o papel que desempenham a mediação social e as competências digitais de pais e filhos em estratégias emergentes de mediação parental que maximizem oportunidades e minimizem riscos. Considerando a preocupação com os riscos digitais, os resultados da análise de respostas parentais, recolhidas em cinco países europeus, permitiram aos autores distinguir entre uma mediação restritiva, marcada por interdições, e uma mediação que os autores designam como capacitante (enabling mediation). Esta, não ignorando a existência dos riscos para as crianças, procura assegurar um acompanhamento próximo, promover um ambiente onde a criança esteja à vontade para iniciar conversas sobre o que a preocupa e onde adquira competências digitais a fim de maximizar oportunidades.

No que se refere a crianças mais novas, a mediação capacitante reclama pais digitalmente competentes na combinação da mediação ativa do uso da internet e na mediação para a sua segurança: pais que incorporam o diálogo, o exemplo e outras práticas sociais com restrições técnicas e monitorização.

Foram estes conceitos de mediação que orientaram a análise aos fatores - do lado das crianças e do lado dos pais - que intervêm na mediação parental nas famílias portuguesas com crianças mais novas que fazem uso da internet. Antes de apresentarmos o modo como fizemos essa análise e os resultados a que chegámos, vejamos traços do contexto português, na próxima secção. 


\section{Famílias portuguesas e o digital}

Mais tarde do que no norte europeu e com diferenças entre gerações, as famílias portuguesas foram entrando no digital na primeira década do novo século: em 2009 mais de metade da população com mais de 16 anos fazia uso da internet. Desde 2002 que, nas estatísticas, a liderança pertence ao grupo mais jovem (16-25 anos), atingindo hoje a quase totalidade deste grupo etário (INE, 2015). Contudo, pouco se sabe relativamente a acessos por grupos etários abaixo dessa idade, que começam a usar esses meios num contexto em rápida mutação.

O inquérito "EU Kids Online", de 2010, realizado em 25 países europeus a crianças e jovens (9-16 anos) e seus pais, revelou que em países do norte europeu os pais usavam mais a internet do que os filhos, ao contrário do que se passava nos países do leste e do sul, evidenciando diferentes vagas de penetração do digital nas respetivas sociedades. Em Portugal, cerca de $40 \%$ dos pais não usava esse meio e apenas um terço fazia um uso diário ou quase todos os dias (Ponte et al., 2012). Era o segundo lugar mais baixo, apenas à frente da Turquia (Livingstone et al., 2011).

Como a pesquisa tem demonstrado, condições de não uso ou de uso esporádico da internet por parte dos pais influenciam modos como consideram os riscos da internet e como intervêm na sua mediação (Clark, Demont-Heinrich e Scott, 2005; Paus-Hasebrink et al., 2013). Importa também ter em conta as preocupações parentais para com os filhos, que se alicerçam em valores culturais e na própria agenda noticiosa com muito mais atenção a riscos do que a oportunidades associadas ao uso de tecnologias.

Em países do sul europeu, o protecionismo é uma prática parental que contrasta com a promoção de uma maior autonomia, mais presente no norte europeu (Helsper et al., 2013). Numa análise longitudinal aos resultados portugueses no European Values Survey, Almeida e Ramos (2018) destacam a hegemonia na transmissão a filhos de valores que facilitem a sua integração e conformidade com quadros de vida existentes, mais do que o incentivo a que sejam criativos, autónomos e determinados. Ainda que a responsabilidade surja como o principal valor a transmitir, tem sido identificada a tendência de os pais considerarem que os seus filhos são "ainda demasiado novos para...", numa lógica de infantilização e de não responsabilização (Ponte e Simões, 2009; Rocha e Ferreira, 2016).

No inquérito "EU Kids Online" de 2010, os pais portugueses lideravam nas preocupações com conteúdos desadequados e com contactos por parte de desconhecidos na internet, duplicando a média europeia (Livingstone et al., 2012). Em linha com esse resultado, a mediação social associada a restrições e a acompanhamento aparecia com valores elevados, coincidindo as respostas de pais com as de filhos. Os pais portugueses lideravam também na mediação restritiva (proibições) e apresentavam valores dos mais elevados em formas de mediação ativa (conversar com os filhos, sentar-se ao lado deles quando estavam a fazer uso, fazer atividades em conjunto...). Por outro lado, estavam abaixo da média europeia na mediação ativa para usos seguros da internet (ajudar em caso de dificuldade, explicar porque certos sites são bons ou maus, sugerir formas de comportamento...), na monitorização (verificar sites visitados, ver que contactos foram adicionados 
nas redes ou as mensagens trocadas...) e na mediação técnica (usos de software antivírus e de controlos parentais sobre tempo e conteúdos).

Como é que a nova geração de pais, mais digitais, está a realizar a mediação de ecrãs com os seus filhos mais novos? Esta foi uma das perguntas orientadoras do primeiro estudo nacional, "Crescendo entre Ecrãs. Usos de Meios Eletrónicos por Crianças (3-8 Anos)", realizado em 2016 para a Entidade Reguladora da Comunicação Social.

O estudo combinou um inquérito nacional, cujos resultados aqui analisamos, com observação de contextos domésticos de uso de meios digitais por crianças destas idades (Ponte et al., 2017). A decisão de incluir ecrãs televisivos e dispositivos digitais (como tablets, smartphones, computadores ou consolas) permitiu situar, a partir das respostas dos pais, a proporção de crianças destas idades que fazem uso de jogos digitais e da internet. Enquanto todos declararam que os filhos viam televisão, metade referiram que aqueles jogavam jogos digitais e $38 \%$ referiram que usavam a internet, numa variação marcada pela idade: $22 \%$ das crianças entre os $3 \mathrm{e}$ os 5 anos; $62 \%$ entre os 6 e os 8 anos.

Três em quatro pais que reportaram que os seus filhos fazem uso da internet consideram que esse uso é bom para o desenvolvimento global da criança; dois em três destacam o seu contributo para o desempenho escolar. Ao mesmo tempo, sete em dez exprimem preocupação com conteúdos não adequados e seis em dez referem preferir que a criança esteja ocupada com outras atividades. Estes resultados com ambivalências sugerem a consciência de que o uso da internet nestas idades proporciona simultaneamente oportunidades e riscos. Vejamos então como estes pais referem a sua mediação parental.

\section{Metodologia e descrição dos clusters}

As perguntas do inquérito foram adaptadas de questionários sobre estas idades aplicados noutros países europeus (Ofcom, 2015; Nikken e Schols, 2015). O inquérito realizou-se entre junho e julho de 2016, junto de uma amostra nacional representativa de agregados familiares com crianças de idades compreendidas naquele intervalo etário, através de um procedimento de amostragem aleatório por random route. A amostra nacional, composta por 656 indivíduos, pretendeu assegurar diversidade geográfica e de origem social das famílias, como se pode ver no relatório final (Ponte et al., 2017).

Foram aplicados dois questionários, um destinado aos pais ou representantes legais da criança, e outro para ser respondido por crianças de 6 a 8 anos.

O questionário aos pais cobriu todos os tópicos do estudo, incluindo questões sobre os filhos (3-8 anos): perguntas sobre ambiente com média e equipamentos digitais existentes em casa e seu acesso/posse pela criança; televisão (conteúdos e escolhas/preferências, mediação e considerações parentais sobre o seu uso); jogos eletrónicos (frequência de uso, equipamentos, preferências e jogar em conjunto); e internet (usos e atividades pela criança, mediações e considerações parentais sobre o seu uso); incluiu ainda informação demográfica sobre os próprios usos dos pais e 
Quadro 1 Dimensões, variáveis e categorias utilizadas na análise

\begin{tabular}{|c|c|c|c|}
\hline Dimensões & Variáveis & Categorias & $\%$ \\
\hline \multirow{3}{*}{$\begin{array}{l}\text { Iniciativa da criança em } \\
\text { falar com os pais (IC) }\end{array}$} & IC1- Falar sobre o que faz & $\begin{array}{l}\text { Sim } \\
\text { Não }\end{array}$ & $\begin{array}{l}67 \\
33\end{array}$ \\
\hline & IC2 - Falar sobre alguma coisa que a perturbou & $\begin{array}{l}\text { Sim } \\
\text { Não }\end{array}$ & $\begin{array}{l}25 \\
75\end{array}$ \\
\hline & IC3 - Pedir conselhos sobre como agir & $\begin{array}{l}\text { Sim } \\
\text { Não }\end{array}$ & $\begin{array}{l}32 \\
68\end{array}$ \\
\hline \multirow{5}{*}{$\begin{array}{l}\text { Frequência de formas de } \\
\text { mediação social ativa por } \\
\text { parte dos pais (MSA) }\end{array}$} & MSA1 - Falar sobre o que a criança faz & $\begin{array}{l}\text { Nunca } \\
\text { Ocasionalmente } \\
\text { Frequentemente }\end{array}$ & $\begin{array}{l}10 \\
58 \\
32\end{array}$ \\
\hline & $\begin{array}{l}\text { MSA2 - Incentivar a explorar e aprender coisas } \\
\text { novas }\end{array}$ & $\begin{array}{l}\text { Nunca } \\
\text { Ocasionalmente } \\
\text { Frequentemente }\end{array}$ & $\begin{array}{r}28 \\
66 \\
6\end{array}$ \\
\hline & MSA3 - Sentar-se ao lado da criança & $\begin{array}{l}\text { Nunca } \\
\text { Ocasionalmente } \\
\text { Frequentemente }\end{array}$ & $\begin{array}{l}10 \\
45 \\
45\end{array}$ \\
\hline & MSA4 - Fazer atividades com a criança & $\begin{array}{l}\text { Nunca } \\
\text { Ocasionalmente } \\
\text { Frequentemente }\end{array}$ & $\begin{array}{l}29 \\
54 \\
28\end{array}$ \\
\hline & $\begin{array}{l}\text { MSA5 - Ajudá-la quando tem dificuldades } \\
\text { em fazer/ encontrar alguma coisa }\end{array}$ & $\begin{array}{l}\text { Nunca } \\
\text { Ocasionalmente } \\
\text { Frequentemente }\end{array}$ & $\begin{array}{l}22 \\
46 \\
33\end{array}$ \\
\hline \multirow{4}{*}{$\begin{array}{l}\text { Frequência de formas de } \\
\text { mediação para a segurança } \\
\text { por parte dos pais (MSG) }\end{array}$} & $\begin{array}{l}\text { MSG1 - Sugerir maneiras de usar internet com } \\
\text { segurança }\end{array}$ & $\begin{array}{l}\text { Nunca } \\
\text { Ocasionalmente } \\
\text { Frequentemente }\end{array}$ & $\begin{array}{l}24 \\
53 \\
22\end{array}$ \\
\hline & $\begin{array}{l}\text { MSG2 - Falar sobre o que fazer se algo a } \\
\text { incomodar }\end{array}$ & $\begin{array}{l}\text { Nunca } \\
\text { Ocasionalmente } \\
\text { Frequentemente }\end{array}$ & $\begin{array}{l}27 \\
54 \\
20\end{array}$ \\
\hline & $\begin{array}{l}\text { MSG3 - Explicar porque é que alguns sites são } \\
\text { adequados ou inadequados }\end{array}$ & $\begin{array}{l}\text { Nunca } \\
\text { Ocasionalmente } \\
\text { Frequentemente }\end{array}$ & $\begin{array}{l}26 \\
48 \\
27\end{array}$ \\
\hline & MSG4 - Ajudar a lidar com algo que o incomodou & $\begin{array}{l}\text { Nunca } \\
\text { Ocasionalmente } \\
\text { Frequentemente }\end{array}$ & $\begin{array}{l}36 \\
48 \\
16\end{array}$ \\
\hline \multirow{6}{*}{$\begin{array}{l}\text { Iniciativa dos pais em } \\
\text { conversar sobre segurança } \\
\text { online (SEC) }\end{array}$} & $\begin{array}{l}\text { SEC1 - Encontrar conteúdos/ websites } \\
\text { inadequados para a idade }\end{array}$ & $\begin{array}{l}\text { Sim } \\
\text { Não }\end{array}$ & $\begin{array}{l}45 \\
55\end{array}$ \\
\hline & SEC2 - Acreditar em tudo o que vê/ lê & $\begin{array}{l}\text { Sim } \\
\text { Não }\end{array}$ & $\begin{array}{l}53 \\
47\end{array}$ \\
\hline & SEC3 - Cyberbullying & $\begin{array}{l}\text { Sim } \\
\text { Não }\end{array}$ & $\begin{array}{l}32 \\
68\end{array}$ \\
\hline & $\begin{array}{l}\text { SEC4 - Publicar informações e fotografias } \\
\text { pessoais }\end{array}$ & $\begin{array}{l}\text { Sim } \\
\text { Não }\end{array}$ & $\begin{array}{l}41 \\
59\end{array}$ \\
\hline & SEC5 - Apanhar vírus, trojans, spyware & $\begin{array}{l}\text { Sim } \\
\text { Não }\end{array}$ & $\begin{array}{l}37 \\
63\end{array}$ \\
\hline & SEC6 - Contactar com estranhos & $\begin{array}{l}\text { Sim } \\
\text { Não }\end{array}$ & $\begin{array}{l}53 \\
47\end{array}$ \\
\hline $\begin{array}{l}\text { Uso de formas de } \\
\text { mediação técnica (MT) }\end{array}$ & MT - Uso de ferramentas digitais & $\begin{array}{l}\text { Sim } \\
\text { Não }\end{array}$ & $\begin{array}{l}14 \\
86\end{array}$ \\
\hline
\end{tabular}


Quadro 2 Análise de correspondências múltiplas: padrões de mediação parental da internet

\begin{tabular}{|c|c|c|}
\hline Dimensão 1: mediação indireta & Negativo & Positivo \\
\hline \multirow{3}{*}{ Iniciativa da criança em falar com os pais (IC) } & IC1.S & IC1.N \\
\hline & IC2.S & IC2.N \\
\hline & IC3.S & IC3.N \\
\hline \multirow{6}{*}{$\begin{array}{l}\text { Iniciativa dos pais em conversar sobre segurança online } \\
\text { (SEC) }\end{array}$} & SEC1.S & SEC1.N \\
\hline & SEC2.S & SEC2.N \\
\hline & SEC3.S & SEC3.N \\
\hline & SEC4.S & SEC4.N \\
\hline & SEC5.S & SEC5.N \\
\hline & SEC6.S & SEC6.N \\
\hline Uso de formas de mediação técnica (MT) & MT.S & MT.N \\
\hline Dimensão 2: mediação ativa & Negativo & Positivo \\
\hline \multirow{3}{*}{$\begin{array}{l}\text { Frequência de formas de mediação social ativa por parte } \\
\text { dos pais (MSA) }\end{array}$} & MSA3.N; MSA3.F & MSA3.O \\
\hline & MSA4.N; MSA4.F & MSA4.O \\
\hline & MSA5.N; MSA5.F & MSA5.O \\
\hline \multirow{4}{*}{$\begin{array}{l}\text { Frequência de formas de mediação para a segurança por } \\
\text { parte dos pais (MSG) }\end{array}$} & MSG1.N; MSG1.F & MSG1.0 \\
\hline & MSG2.N; MSG2.F & MSG2.O \\
\hline & MSG3.N; MSG3.F & MSG3.O \\
\hline & MSG4.N; MSG4.F & MSG4.O \\
\hline
\end{tabular}

Nota: As variáveis de mediação ativa sobre "falar sobre o que o filho faz" (MSA1) e "incentivar a explorar e aprender coisas novas" (MSA2) são transversais às duas dimensões

a sua experiência relativamente ao início do uso da internet. O questionário destinado a crianças (6-8 anos) compreendeu módulos simplificados de tópicos sobre acessos, usos e mediações (com pais, irmãos, amigos), bem como perguntas abertas sobre programas de televisão e atividades digitais.

Esta análise, sobre o questionário respondido pelos pais, reflete os resultados sobre as mediações sociais e sobre mediações para a segurança, incidindo sobre um recorte específico da amostra, nomeadamente, as respostas dos pais que declararam que os seus filhos fazem uso da internet. Este recorte reduz a amostra inicial para $38 \%$ dos casos $(\mathrm{N}=251)$, como referimos.

De maneira a identificar padrões de mediação parental foram consideradas 19 variáveis incluídas no questionário dos pais (cf. quadro 1), que contemplam: (1) a iniciativa da criança em falar com os pais; (2) a frequência de formas de mediação social ativa do uso da internet por parte dos pais; (3) a frequência de formas de mediação ativa para a segurança na internet por parte dos pais; (4) a iniciativa dos pais em conversar sobre segurança online; (5) o uso de restrições técnicas.

Da análise de correspondências múltiplas ao conjunto das 19 variáveis realizada com recurso ao programa IBM SPSS (versão 23), resultou a identificação de três padrões de mediação, organizados por duas dimensões. A primeira dimensão remete para a existência (ou não) de uma mediação indireta através de conversas sobre internet, iniciadas tanto pelos pais como pelas crianças, ou pelo uso de ferramentas técnicas. A segunda dimensão diz respeito à 


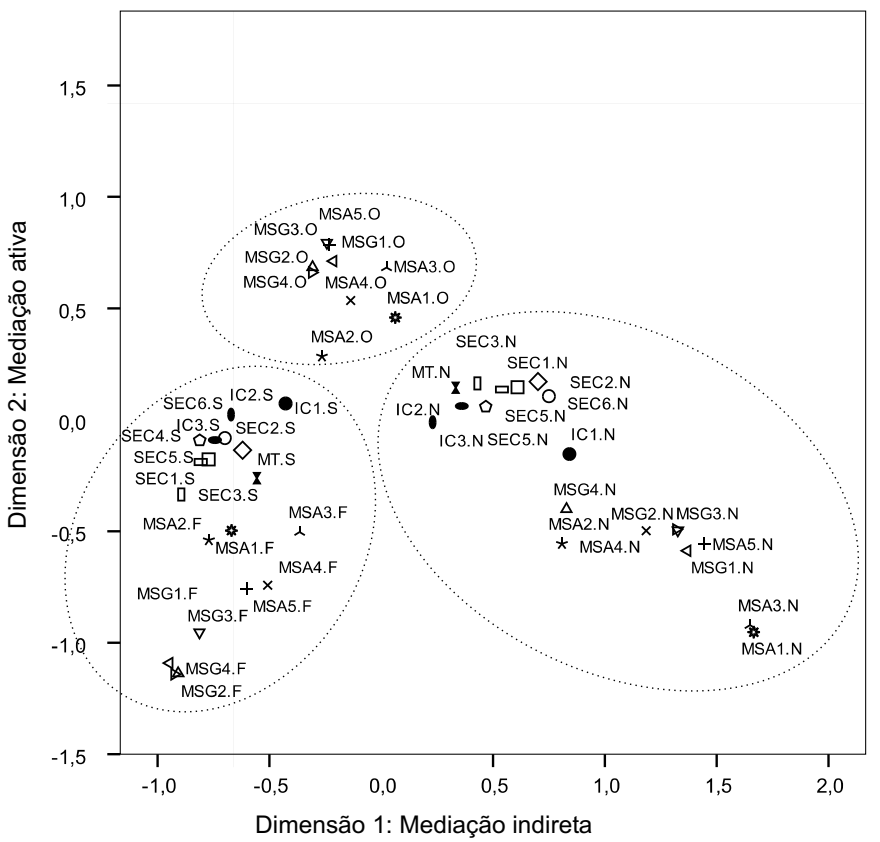

- IC1- Falar sobre o que faz - IC2 - Falar sobre alguma
coisa que o perturbou

- IC3 - Pedir conselhos sobre

- como agir

* MSA1 - Falar sobre o que o

MSA2 - Incentivar a explora

e aprender coisas novas

MSA3 - Sentar-se ao lado

da criança

$\times$ MSA4 - Fazer atividades MSA5 - Ajud́-

MSA5 - Ajudá-la quando ter dificuldades em fazer/ MSG1- Sugerir maneiras de $\triangleleft$ usar internet com

segurança

$\triangle$ MSG2 - Falar sobre o que MSG3 - Explicar porque

$\nabla$ que alguns sites são

adequados ou inadequados $\triangle$ MSG4 - Ajudar a lidar com

algo que o incomodou

MT-Uso de ferramentas SEC1

SEC1 - Encontrar inadequados para a idade $\checkmark$ SEC2 - Acreditar em tudo o

$\checkmark$ SEC3 - Ciberbullying

- SEC4 - Publicar informaçõe

SEC5 - Apanhar víus,

SEC5 - Apanhar virus,
trojans, spyw are

SEC6 - Contactar com

Dimensão 1: Mediação indireta

Figure 1 Padrões de mediação parental da internet

intensidade da frequência de uma mediação ativa, quer seja sobre segurança online ou não.

O quadro 2 sintetiza as variáveis que mais distinguem essas duas dimensões e o posicionamento das suas categorias (no anexo encontram-se as medidas de discriminação e as contribuições das variáveis). Tal como sugerido em Carvalho (2008), a interpretação das dimensões também teve em conta a leitura das medidas de quantificação e contribuição das categorias das variáveis.

Como se pode verificar, na primeira dimensão opõem-se as categorias de existência ou inexistência de conversas ou de uso das ferramentas técnicas; na segunda dimensão, opõem-se os extremos da escala de frequência das formas de mediação ativa ou mediação ativa da segurança (categorias nunca e frequentemente) com a categoria média (ocasionalmente) dessas mesmas variáveis.

O plano, definido por essas duas dimensões e representado na figura 1, evidencia diferentes configurações de associações entre categorias, sugerindo três tipos de práticas de mediação parental da internet. Um primeiro tipo identifica práticas de mediação ativa frequente, com conversas sobre segurança iniciadas pelos pais, conversas sobre internet iniciadas pelas crianças, bem como o uso de ferramentas digitais por parte dos pais. Um segundo tipo distingue-se pela ausência dessas práticas. Estes dois tipos surgem em contraponto, emergindo em polos opostos da dimensão 1 . O terceiro tipo caracteriza-se por práticas moderadas de mediação ativa. 
A partir destes resultados, uma análise de clusters $^{1}$ permitiu classificar cada respondente num de três tipos, que designámos por implicados, intermitentes e desengajados.

O grupo dos mediadores implicados $(\mathrm{N}=65,26 \%$ dos inquiridos) corresponde ao padrão da mediação ativa frequente combinando conversas e controlos técnicos. O grupo numericamente maior, a que chamamos mediadores intermitentes $(\mathrm{N}=128,51 \%)$, corresponde a uma mediação ativa moderada, portanto com um menor grau de envolvimento na orientação digital da criança. Finalmente, o último grupo, que cobre $21 \%$ da amostra (58 indivíduos), diz respeito aos mediadores desengajados (ou "não mediadores"), aqueles que não realizam práticas de mediação.

Analisaram-se estes clusters por relação às crianças (idade, sexo, viverem ou não com irmãos) e aos pais (distinção entre pai ou mãe; características socioeconómicas do agregado; escolaridade; experiência digital; considerações em relação à internet e competências digitais das crianças). Nos próximos pontos, apresentam-se e discutem-se esses resultados.

\section{Resultados}

A leitura inicial dos resultados parte de uma análise descritiva dos clusters criados tendo em conta o seu cruzamento com variáveis de caracterização, ${ }^{2}$ número de competências das crianças em relação à internet, uso desta pelos pais e perceções e atitudes parentais em relação à mesma (cf. quadro 3). Esta leitura permite-nos definir um retrato descritivo dos grupos com base nos resultados dos quadrantes fatoriais da figura 1 . Sobressai a importância das variáveis de caracterização consideradas para interpretar os grupos (clusters) criados, permitindo compreender de que modo as práticas em questão variam de acordo com estas variáveis e perceber em que medida as especificidades dos grupos identificados se associam a diferentes perfis de crianças e respetivos pais.

\section{Mediadores implicados}

Cerca de um quarto dos pais situa-se neste grupo. O que mais caracteriza esta mediação, relativamente às outras duas, são práticas como conversar com frequência com a criança sobre questões de segurança (MSG1 MSG2 MSG3 MSG4), ajudá-la quando tem dificuldade e fazer usos em conjunto (MSA5 MSA4), incentivá-la a

1 A análise de clusters foi realizada a partir dos scores dos objetos obtidos pela análise de correspondências múltiplas. Tendo-se decidido na análise exploratória pela definição de três grupos (foram usados vários métodos, mas o método "ligação entre grupos" foi o que apresentou resultados mais consistentes e claros), a classificação dos inquiridos foi refinada com recurso ao método não hierárquico K-means.

2 Para cada cruzamento, foi aplicado o teste do Qui-quadrado. A significância do mesmo surge indicada no final da tabela para cada cruzamento 
Quadro 3 Clusters segundo variáveis de caracterização, competências atribuídas às crianças e atitudes em relação à internet

\begin{tabular}{|c|c|c|c|c|}
\hline$\%$ & $\begin{array}{c}\text { Mediadores } \\
\text { intermitentes } \\
(N=128)\end{array}$ & $\begin{array}{c}\text { Mediadores } \\
\text { implicados } \\
(N=65)\end{array}$ & $\begin{array}{c}\text { Mediadores } \\
\text { desengajados } \\
(N=58)\end{array}$ & $\begin{array}{c}\text { Total } \\
(\mathrm{N}=256)\end{array}$ \\
\hline \multicolumn{5}{|l|}{ Sexo da criança* } \\
\hline Rapaz & 52 & 55 & 45 & 51 \\
\hline Rapariga & 48 & 45 & 55 & 49 \\
\hline \multicolumn{5}{|l|}{ Idade da criança****} \\
\hline $3-5$ anos & 35 & 14 & 60 & 35 \\
\hline $6-8$ anos & 65 & 86 & 40 & 65 \\
\hline \multicolumn{5}{|l|}{ Inquirido* } \\
\hline Pai & 30 & 20 & 24 & 26 \\
\hline Mãe & 70 & 80 & 76 & 74 \\
\hline \multicolumn{5}{|c|}{ Criança vive com irmãos* } \\
\hline Sim & 35 & 34 & 33 & 34 \\
\hline Não & 65 & 66 & 67 & 66 \\
\hline \multicolumn{5}{|c|}{ Estatuto socioeconómico do agregado** } \\
\hline Baixo & 27 & 14 & 34 & 25 \\
\hline Médio & 47 & 49 & 41 & 46 \\
\hline Alto & 27 & 37 & 24 & 29 \\
\hline \multicolumn{5}{|c|}{ Escolaridade do inquirido ${ }^{* * *}$} \\
\hline Até $6^{\circ}$ ano & 19 & 8 & 17 & 16 \\
\hline $9^{\circ}$ ano & 28 & 14 & 22 & 23 \\
\hline $12^{\circ}$ ano & 29 & 43 & 43 & 36 \\
\hline Médio/ superior & 24 & 35 & 17 & 25 \\
\hline \multicolumn{5}{|l|}{ Uso da Net pais ${ }^{(1) *}$} \\
\hline Nativos & 44 & 43 & 30 & 41 \\
\hline Imigrantes & 40 & 41 & 59 & 45 \\
\hline Não utilizadores & 16 & 16 & 11 & 15 \\
\hline \multicolumn{5}{|l|}{$\mathrm{N}^{0}$ competências $^{(2) * * *}$} \\
\hline Uma & 52 & 26 & 75 & 46 \\
\hline Duas & 23 & 28 & 15 & 24 \\
\hline Três & 15 & 21 & 10 & 16 \\
\hline Quatro e mais & 10 & 25 & 0 & 14 \\
\hline \multicolumn{5}{|c|}{ Net como meio de aprendizagem ${ }^{(3)_{\star * \star *}}$} \\
\hline Discorda & 2 & 5 & 3 & 3 \\
\hline Neutro & 18 & 8 & 36 & 20 \\
\hline Concorda & 80 & 88 & 60 & 78 \\
\hline \multicolumn{5}{|c|}{ Prefere que a criança faça outra coisa* } \\
\hline Discorda & 10 & 6 & 4 & 8 \\
\hline Neutro & 35 & 32 & 26 & 33 \\
\hline Concorda & 54 & 62 & 70 & 60 \\
\hline \multicolumn{5}{|c|}{ Net como meio de riscos ${ }^{\star * *}$} \\
\hline Discorda & 12 & 14 & 19 & 14 \\
\hline Neutro & 39 & 22 & 45 & 36 \\
\hline Concorda & 50 & 65 & 36 & 50 \\
\hline \multicolumn{5}{|l|}{ Net como vício* } \\
\hline Discorda & 64 & 74 & 59 & 65 \\
\hline Neutro & 20 & 11 & 18 & 17 \\
\hline Concorda & 16 & 15 & 23 & 17 \\
\hline
\end{tabular}

${ }^{*}$ Não significativo; ${ }^{* *} p<0,05 ;{ }^{* * *} p<0,01 ;{ }^{* * * *} p<0,001$

(1) Os 'nativos digitais' tinham menos de 20 anos quando iniciaram o uso da internet; os 'imigrantes' mais de 20 anos.

(2) Número de competências das crianças indicado pelos pais (por exemplo: fazer download de conteúdos ou aplicações, localizar aplicações ou encontrar conteúdos, etc.).

(3) As considerações dos pais sobre a internet resultaram do seu grau de concordância relativamente a conjuntos de afirmações, como por exemplo, "O meu filho tem dificuldade em deixar de usar a internet por sua livre vontade" (no caso da consideração da internet como vício). 
explorar e a aprender coisas novas na internet, sentar-se ao lado e conversar com ela sobre o que faz (MSA2 MSA1 MSA3) e sobre cyberbullying (SEC3). É o único grupo associado ao uso de ferramentas digitais para promoção de segurança (MT) e à iniciativa da criança em falar com os pais sobre vivências digitais que realiza, sobre o que a incomodar ou pedir conselhos (IC1, IC2, IC3).

Tendo em conta as crianças, este tipo de mediação é muito mais comum com as mais velhas e é mais apontada no caso de rapazes. Viver ou não com irmãos não afeta os resultados.

Verifica-se uma relação direta com o estatuto socioeconómico do agregado e com o grau de escolaridade dos pais: os pais com ensino secundário ou superior mais que duplicam os que têm nove anos de escolaridade ou menos. As mães superam os pais e a diferença de experiência digital (serem nativos ou imigrantes digitais ou não utilizarem) condiciona ligeiramente a adoção desta mediação.

Este é o cluster com menores variações nas respostas ao número de competências digitais dos filhos, sendo aquele em que mais pais (cerca de um quarto) lhes reconhecem quatro ou mais competências. É também aquele onde há maior concordância sobre as potencialidades da internet como meio de aprendizagem $(88 \%$ concordam, $5 \%$ discordam, e $8 \%$ não têm opinião) e simultaneamente como meio de riscos ( $65 \%$ concordam, $14 \%$ discordam e $22 \%$ não têm opinião), justificando talvez assim o seu empenho no acompanhamento próximo da experiência digital dos filhos. É ainda o cluster onde mais pais discordam com a potencialidade de vício da internet, sugerindo uma situação sob o seu controlo.

Esta mediação, que se aproxima do conceito de enabling mediation que fomos buscar a Livingstone et al. (2017), confirma a atenção aos riscos e concentra-se nos esforços de capacitação para desenvolver competências digitais nas crianças, acreditando no seu potencial de aprendizagem.

\section{Mediadores desengajados}

Um pouco menos de um quarto dos pais situa-se neste cluster. Este grupo define-se por ser o que menos fala com a criança sobre o que ela faz na internet (MSA1) ou menos se senta próximo dela quando a está a usar (MSA3). Distingue-se, ainda, por não ajudar a criança a resolver problemas (MSA5), não dar sugestões de segurança (MSG1), não falar sobre situações que a possam ter incomodado (MSG2), não explicar a adequação de determinados sites (MSG3), nem fazer usos em conjunto (MSA4). A ausência de mediação sobre as práticas referidas ocorre maioritariamente nos casos dos pais com crianças mais novas. As diferenças são estatisticamente significativas e sugerem que a menor idade (neste caso, entre os 3 e os 5 anos) é associada a uma dispensabilidade de orientação. Ao contrário da anterior, ocorre mais com raparigas do que com rapazes. A criança viver ou não com irmãos, mais uma vez, parece não ter influência.

É nesta não mediação que se encontram mais agregados com nível socioeconómico baixo. Está bastante mais presente entre os pais com habilitações médias, entre o 9.. $.^{\circ} 12 .{ }^{\circ}$ anos do que entre pais menos escolarizados (6.. ano) ou com o nível superior. Os pais "imigrantes digitais", aqueles que começaram a usar a internet depois dos seus 20 anos, são os que mais se distinguem nesta não mediação. 
Sem surpresa, dado o baixo acompanhamento, dois terços dos pais reconhecem apenas uma competência digital à criança, não indo além da identificação máxima de três. Ao contrário dos do grupo dos mediadores implicados, este grupo revela-se menos seguro acerca das potencialidades educativas da internet: mais de um terço não toma posição sobre se é ou não um meio de aprendizagem. São os pais que mais dizem preferir que a criança faça outra coisa em vez de estar na internet e que mais consideram que esta pode gerar vício. Por outro lado, é o grupo que menos reconhece a potencialidade de riscos da internet - $36 \%$ concordam e $45 \%$ não tomam posição - sendo este resultado estatisticamente significativo.

Estes resultados, que parecem estar associados a pais mais velhos do que os do grupo anterior, sugerem como que uma demissão com as práticas digitais que proporcionam aos filhos, nestas idades. A tecnologia parece figurar como um recurso inevitável do tempo presente, um "brinquedo" a juntar a outros, que permite a realização de atividades, sem que se tenha em conta tanto as suas potencialidades educativas ou os riscos a que frequentemente é associada. Parece existir consonância com discursos vigentes, como o das crianças como "nativos digitais" ou da "idade da inocência" que através das suas práticas as famílias parecem preservar (ver Ponte et al., 2017).

\section{Mediadores intermitentes}

Cerca de metade dos pais situa-se neste grupo, que se define mais pela positiva (agir, fazer alguma coisa) do que pela negativa (não agir). O que distingue este grupo relativamente ao dos pais implicados é a menor intensidade desse agir, que é ocasional, um fazer às vezes. De acordo com a figura 1 , este cluster concretiza um padrão de mediação sobretudo constituído por práticas de mediação social ativa (MSA1 MSA2 MSA3 MSA4 MSA5) e sobre segurança (MSG1 MSA2 MSG3 MSG4).

As variações por idade da criança são menos evidentes que no grupo dos mediadores implicados, embora continuem mais frequentes entre as crianças mais velhas. Há diferenças mínimas por sexo da criança.

Este tipo de mediação é mais elevado entre agregados com nível socioeconómico médio, reproduzindo o padrão geral da amostra. Por escolaridade, ocorre mais com pais com 9. ano ou 12.ำ ano, mas é também transversal. O grupo que mais contribui é o dos pais nativos digitais, tal como nos mediadores empenhados.

Metade destes pais reconhecem apenas uma competência digital da criança. O número de competências reconhecidas decresce, numa expressão menos acentuada do que a dos mediadores desengajados. Este grupo de pais é semelhante aos mediadores implicados no que respeita à concordância com as potencialidades da internet como meio de aprendizagem e às considerações sobre riscos e vício, embora com variações menores entre as posições. $\mathrm{O}$ mais expressivo contraste com os outros dois grupos no que às considerações diz respeito observa-se na menor percentagem de pais deste grupo que concordam que outra atividade seria preferível. 
A intermitência vai ao encontro de uma aparente menor consistência nas orientações, a uma certa dificuldade de tomar posição. Neste grupo, o mais numeroso, parece impor-se a ideia da "internet, porque sim", numa expressão de confiança no determinismo tecnológico.

Influência de fatores associados a crianças e a pais

Numa leitura global do quadro 3, destacamos de seguida as tendências dos fatores relacionados com crianças e com pais.

Tendo como ponto de partida a criança, a idade diferencia claramente as formas de mediação parental que se opõem - mediadores implicados versus mediadores desengajados. Tais diferenças também se observam se considerarmos o sexo da criança: há mais pais com filhas que praticam mediações desengajadas, embora as diferenças sejam menos expressivas. Viver ou não com irmãos parece não ter influência na mediação parental.

Relativamente aos pais, não existem diferenças significativas por género, embora haja mais pais entre os mediadores intermitentes e mais mães entre os mediadores engajados. A experiência digital dos pais também distingue principalmente mediadores implicados e intermitentes face aos desengajados, onde há uma maior percentagem de pais que começaram a usar a internet depois dos 20 anos.

A escolaridade dos pais e o estatuto socioeconómico do agregado aparecem como os indicadores mais significativos, destacando sobretudo os mediadores implicados. Estes resultados confirmam questões de desigualdade perante diferentes recursos culturais e económicos.

\section{Discussão}

Com base no primeiro estudo nacional sobre como as crianças mais novas estão a crescer entre ecrãs, este artigo focou-se nas formas de mediação parental quando essas crianças fazem uso de meios digitais. O primeiro resultado que se destacou é que, segundo os pais, é elevado o número das crianças que não fazem esse uso. Importa destacar que estudos de tipo qualitativo realizados junto de famílias portuguesas (Dias e Brito, 2016), ao serem tratados de forma generalizada pelos média, alimentaram a ideia de que a larga maioria de crianças destas idades utilizavam meios móveis, como tablets e smartphones.

Não foi essa a tendência encontrada no estudo "Crescendo entre Ecrãs", cujos resultados globais quantitativos aqui foram apresentados. Evidenciou-se um contraste entre lares tecnologicamente equipados, na sua larga maioria, com meios móveis e o número relativamente baixo de pais (38\%) que declararam que a criança usava a internet. Dessa resposta, que sugere uma intervenção restritiva, podem não estar ausentes associações a juízos de valor sobre o que é socialmente desejável, nomeadamente a ideia do que constitui ser "bom pai" ou "boa mãe", num contexto - em Portugal como noutros países - onde as referências nos média a perigos dos novos ecrãs superam largamente as que 
destacam potencialidades ou as que equilibram os dois polos (Blum-Ross e Livingstone, 2015).

Pode-se dar também o caso de a pergunta - traduzida de questionários feitos no ano anterior, 2015, no Reino Unido - ter sido interpretada em 2016 pelos pais portugueses com base no conceito tradicional de "ir à internet": as formas de aceder comuns antes do boom dos ecrãs táteis e das aplicações com ícones, quando havia um indispensável ato de escrita e manejo de teclado para se entrar num determinado ambiente digital. Hoje, ainda antes de saber ler e escrever, uma criança pode "estar na internet" entrando por via de ícones que facilmente reconhece e associa a um serviço específico. A crescente porosidade entre o mundo off-line e o mundo online e a facilidade com que se está na rede quando se vive em lares tecnologicamente equipados tornam necessária a formulação adequada de perguntas (por exemplo, em vez de perguntar se a criança "usa a internet", perguntar se ela faz uso de aplicações como o YouTube).

Foi isso que foi feito na parte qualitativa deste estudo, onde questões que constavam do guião de entrevistas aos pais sobre práticas digitais das crianças ou sobre as suas competências digitais tiveram de ser desdobradas e associadas a situações observadas junto das crianças da casa (Castro et al., 2017; Ponte, Castro e Batista, 2018).

A decisão de incluir todos os ecrãs - e não apenas os ecrãs dos novos meios digitais - permitiu assinalar o "grande ecrã da televisão", que aos olhos dos pais, funciona como uma espécie de um "jardim murado" e seguro de que as crianças destas idades podem usufruir (todos os pais declararam que as crianças veem televisão todos os dias), enquanto os ecrãs digitais Wi-Fi parecem ser vistos como mais inquietantes por vários pais, aspeto confirmado na pesquisa qualitativa (Ponte et al., 2017).

A pesquisa sobre mediações realizada entre o conjunto de pais que declararam que os filhos usavam a internet, e que constitui o foco deste artigo, deve ter em conta o relativamente baixo número de respostas recolhidas.

Um primeiro resultado a destacar é o de, aparentemente, a ideia de que fazer parte da primeira geração de pais que cresceram com a internet iria ter repercussão na mediação parental destas crianças não se ter verificado com a dimensão esperada. Há de facto menos pais "nativos digitais" entre os que praticam uma mediação desengajada, mas as diferenças não são estatisticamente significativas. Mais do que o histórico da idade com que começaram a usar, revelaram ter peso fatores ligados à escolaridade dos pais e ao estatuto socioeconómico do agregado.

Outro resultado interessante é o posicionamento de pais e de mães, os primeiros com maior peso na mediação intermitente do que em qualquer outro grupo e as segundas a surgirem claramente à frente na mediação engajada. Ainda que ambos sejam profissionalmente ativos na sociedade portuguesa, esta diferença corrobora as representações sociais que se atribuem à mãe, sendo esta a ocupar a principal posição como cuidadora e a estar mais presente no quotidiano da criança, e ao pai uma posição mais secundária, por menor disponibilidade de tempo para acompanhar a criança.

O predomínio de uma mediação ativa de baixa intensidade, que designámos mediação intermitente, é outra das principais tendências encontradas. Deve ser 
lida com as devidas cautelas, uma vez que assenta em considerações subjetivas sobre frequência (às vezes, raramente). De certo modo, contrasta com anteriores valores reportados por pais portugueses, de elevada preocupação com riscos digitais, encontrados em resultados sobre mediação parental junto de pais de crianças mais velhas e também eles utilizadores menos frequentes de meios digitais, como apareceu no inquérito "EU Kids Online" de 2010 (Livingstone et al., 2011). A menor idade da criança pode pesar neste resultado.

É na mediação intermitente que se situam mais de metade dos pais (e mais pais propriamente ditos), percorrendo todos os agregados socioeconómicos. Nestas idades mais novas, parece ser uma mediação associada a uma confiança nas potencialidades da internet como meio de entretenimento e de aprendizagem "por $\mathrm{si}^{\prime \prime}$, o que levará a que considerem bem despendido o tempo em que a criança reserva a essa atividade.

Considerando as crianças destas idades como parceiras desta mediação, e tendo presente a importância de adquirirem competências digitais em ambientes de segurança e desde cedo, os resultados apontam que não só a idade faz claramente a diferença, mas também que os rapazes parecem beneficiar de uma mediação mais estimuladora do desenvolvimento das suas competências. Este resultado deve merecer atenção na medida em que pode ir ao encontro da ideia socialmente dominante de que eles têm mais capacidades do que elas na sua relação com a tecnologia.

\section{Notas finais}

Esta tipologia de caracterização de estilos de mediação parental não deve ser lida de uma forma absoluta. Como a pesquisa qualitativa que foi parte deste estudo deu conta, as dinâmicas familiares estão pejadas de paradoxos e de tensões (Castro $e t$ al., 2017). Mesmo assim, os resultados alcançados - que devem ter em conta as limitaçoes associadas ao baixo número de respondentes que constituíram a amostra desta análise, repetimos - apontam tendências que merecem atenção.

O muito baixo uso de ferramentas para a segurança digital continua a ser largamente dominante entre os pais portugueses. Em contextos familiares onde muitas vezes as crianças partilham dispositivos digitais dos pais, a maior segurança conseguida pelo uso de instrumentos de controlo deve merecer uma sensibilização mais eficaz por parte de responsáveis, nomeadamente as próprias indústrias.

A mediação que quantitativamente mais se distingue aponta para uma intervenção ativa, mas esporádica, e para uma forte confiança nas potencialidades da tecnologia "em si", como recurso de aprendizagem e de ocupação de tempos livres. Este é também um ponto a considerar na sensibilização familiar, contrariando a ideia dos "nativos digitais".

O peso expressivo de mediadores desengajados, deixando as crianças entregues a si mesmas e com baixa perceção de que essa experiência comporta riscos, alerta para a necessidade de ter em conta as suas menores competências parentais. 
Importa promover a sua capacitação digital de um modo adequado - o mesmo "figurino" não serve a todos - envolvendo, além da família, ambientes como a escola ou o jardim de infância - onde as crianças comecem desde cedo a serem capacitadas para o exercício da sua cidadania digital pela aquisição de competências digitais fundamentais.

Não deixa de ser desafiante constatar que estes dois tipos de mediação - intermitente e desengajada - tenham sido aqueles onde o lugar de agência da criança - a falar sobre o que faz, o que a perturbou ou a pedir conselhos sobre como agir - tenha permanecido vazio.

Anexo Contribuição das variáveis de caracterização do espaço dos tipos de mediação parental da internet

\begin{tabular}{l|c|c|c|c}
\hline \multirow{2}{*}{ Variáveis } & \multicolumn{3}{c}{ Dimensões } \\
\cline { 2 - 4 } & \multicolumn{2}{|c}{1} & \multicolumn{2}{c}{2} \\
\cline { 2 - 4 } & Discriminação & Contribuição & Discriminação & Contribuição \\
\hline IC1 - A criança falar sobre o que faz & 0,351 & 4,47 & 0,011 & 0,27 \\
IC2 - Falar sobre o que o/a perturbou & 0,150 & 1,92 & 0,000 & 0,01 \\
IC3 - Pedir conselhos & 0,259 & 3,30 & 0,005 & 0,12 \\
AT1 - Pais falam sobre o que faz & 0,421 & 5,37 & 0,288 & 6,94 \\
AT2 - Incentivo & 0,265 & 3,38 & 0,157 & 3,79 \\
AT3 - Companhia & 0,320 & 4,08 & 0,405 & 9,77 \\
AT4 - Co-utilização & 0,338 & 4,31 & 0,351 & 8,45 \\
AT5 - Ajudam a fazer ou a encontrar & 0,582 & 7,41 & 0,528 & 12,73 \\
ATS1 - Sugestão segurança & 0,668 & 8,52 & 0,610 & 14,70 \\
ATS2 - Falar s/ o que fazer c/ incómodo & 0,671 & 8,55 & 0,542 & 13,07 \\
ATS3 - Explicar (in)adequação & 0,643 & 8,19 & 0,595 & 14,34 \\
ATS4 - Ajudar com incómodo & 0,412 & 5,25 & 0,476 & 11,48 \\
SEC1 - Encontrar coisas inadequadas & 0,462 & 5,89 & 0,025 & 0,61 \\
SEC2 - Acreditar em tudo & 0,426 & 5,43 & 0,023 & 0,56 \\
SEC3 - Ciberbullying & 0,378 & 4,81 & 0,053 & 1,29 \\
SEC4 - Publicar informação pessoal & 0,428 & 5,45 & 0,025 & 0,60 \\
SEC5 - Apanhar vírus etc & 0,374 & 4,76 & 0,005 & 0,13 \\
SEC6 - Contactar c/ estranhos & 0,515 & 6,56 & 0,009 & 0,21 \\
MT - Mediação técnica & 0,184 & 2,34 & 0,038 & 0,92 \\
\hline Total & 7,847 & 100,00 & 4,148 & 100,00 \\
\hline Inércia & 0,413 & & 0,218 & \\
\hline
\end{tabular}

\section{Referências bibliográficas}

Almeida, Ana Nunes, e Vasco Ramos (2018), "As crianças nas famílias em Portugal", em Carla Martins e Cristina Ponte (orgs.) Boom Digital? Crianças (3-8 anos) e Ecrãs. Lisboa, Entidade Reguladora para a Comunicação.

Blum-Ross, Alicia, e Sonia Livingstone (2015), "Families and screen time. current advice and emerging research" Media Policy Brief, 17, Londres, LSE.

Carvalho, Helena (2008), Análise Multivariada de Dados Qualitativos. Utilização da Análise de Correspondências Múltiplas com o SPSS, Lisboa, Sílabo. 
Castro, Teresa Sofia, Cristina Ponte, Susana Batista, e Ana Jorge (2017), "Crescendo entre ecrãs: competências digitais de crianças de 3 a 8 anos", Atas do IV Congresso Literacia, Media e Cidadania, Braga, Universidade do Minho.

Chaudron, Stéphane, Manfred Beutel, Martina Cernikova, Veronica Donoso Navarette, Michael Dreier, Ben Fletcher-Watson, Anni-Sofia Heikkilä, Vera Kontríková, Riitta-Liisa Korkeamäki, Sonia Livingstone, Jackie Marsh, Giovanna Mascheroni, Marina Micheli, Daniele Milesi, Kai Müller, Tuula Myllylä-Nygård, Marja Niska, Oxana Olkina, Svenja Ottovordemgentschenfelde, Lydia Plowman, Wannes Ribbens, Janice Richardson, C. Schaack, Vladimir Shlyapnikov, David Šmahel, Galina Soldatova, e Klaus Wölfling (2015), Young Children (0-8) and Digital Technology. A Qualitative Exploratory Study across Seven Countries, JRC 93239/EUR 27052.

Clark, Lynn Schofield (2011), "Parental mediation theory for the digital age", Communication Theory, 21, pp. 323-343.

Clark, Lynn Schofield, Christof Demont-Heinrich, e Webber Scott (2005), "Parents, ICT, and children's prospects for success: interviews along the digital 'access rainbow'", Critical Studies in Media Communication, 22 (5), pp. 409-426.

Dias, Patrícia, e Rita Brito (2016). Crianças (0-8 Anos) e Tecnologias Digitais. Um Estudo Qualitativo Exploratório, Lisboa, Centro de Estudos de Comunicação e Cultura da Católica.

Duerager, Andrea, e Sonia Livingstone (2012), How Can Parents Support Children's Internet Safety?, Londres, LSE, EU Kids Online, ISSN 2045-256X.

Haddon, Leslie, e Jane Vincent (2014), European Children and Their Career's Understanding of Use, Risks and Safety Issues Relating to Convergent Mobile Media. UCSC, Milano: Net Children Go Mobile, http://www.kidsenjongeren.nl/wp-content/uploads/2012/09/NCGM_ QualitativeReport_D4.pdf

Helsper, Ellen Johanna, Veronika Kalmus, Uwe Hasebrink, Bence Sagvari, e Jos de Haan, (2013), Country Classification. Opportunities, Risks, Harm and Parental Mediation, Londres, LSE, EU Kids Online ISSN 2045-256X.

INE (2015). Sociedade da Informação e do Conhecimento - Inquérito à Utilização de Tecnologias, Lisboa, INE.

Livingstone, Sonia, e Ellen Helsper (2008), "Parental mediation and children's Internet use", Journal of Broadcasting \& Electronic Media, 52 (4), pp. 581-599.

Livingstone, Sonia, Leslie Haddon, Anke Görzig, e Kjartan Ólafsson (2011), EU Kids Online II, Final Report, disponível em: http://eprints.lse.ac.uk/39351/

Livingstone, Sonia, Kjartan Ólafsson, Brian O’Neill, e Veronica Donoso (2012), “Towards a better internet for children. findings and recommendations from EU Kids Online to inform the CEO Coalition", disponível em: http://eprints.lse.ac.uk/44213/

Livingstone, Sonia, Giovanna Mascheroni, Michael Dreier, Stephane Chaudron, e Kaat Lagae (2015), How Parents of Young Children Manage Digital Devices at Home. The Role of Income, Education and Parental Style, Londres, LSE, EU Kids Online, ISSN 2045-256X

Livingstone, Sonia, Kjartan Ólafsson, Ellen Johanna Helsper, Francisco Lupiáñez-Villanueva, Giuseppe Alessandro Veltri, e Frans Folkvord (2017), 
"Maximizing opportunities and minimizing risks for children online: the role of digital skills in emerging strategies of parental mediation", Journal of Communication, DOI: 10.1111/jcom.12277.

Nathanson, Amy (1999), "Identifying and explaining the relationship between parental mediation and children's aggression", Communication Research, 26 (2), pp. 124-143.

Nelissen, Sara, e Jan Van den Bulck (2017), "When digital natives instruct digital immigrants: active guidance of parental media use by children and conflict in the family", Information, Communication E Society, DOI: 10.1080/1369118X.2017.1281993.

Nikken, Peter, e Marjon Schols (2015), "How and why parents guide the media use of young children", Journal of Children and Family Studies, 24 (11), pp. 3423-3435.

Ofcom (2015), Children and Parents. Media Use and Attitudes Report, 2015, disponível em: https://www.ofcom.org.uk/research-and-data/media-literacy-research/childrens/childr en-parents-nov-15

Pasquier, Dominique, José Alberto Simões, e Elodie Kredens (2012), “Agents of mediation and sources of safety awareness: a comparative overview", em Sonia Livingstone, Leslie Haddon e Anke Görzig (orgs.), Children, Risk and Safety on the Internet. Research and Policy Challenges in Comparative Perspective, Bristol, The Policy Press, pp. 219-230.

Paus-Hasebrink, Ingrid, Joke Bauwens, Andrea Dureger, e Cristina Ponte (2013), "Internet, parent-child relationship and socio-cultural differences", Journal of Children and Media, 7 (1), pp. 114-132.

Ponte, Cristina, e José Alberto Simões (2009), “Asking parents about children's internet use: comparing findings about parental mediation in Portugal and other European countries", em Sonia Livingstone e Leslie Haddon, Final Conference EU Kids Online, Londres, disponível em:

http://www.fcsh.unl.pt/eukidsonline/docs/Asking\%20parents-FINAL\%20Paper1_ 27-05-09.pdf

Ponte, Cristina, Ana Jorge, José Alberto Simões, e Daniel Cardoso (2012), Crianças e Internet em Portugal, Coimbra, Minerva Coimbra.

Ponte, Cristina, José Alberto Simões, Susana Batista, Teresa Sofia Castro, e Ana Jorge (2017), Crescendo entre Ecrãs. Uso de Meios Eletrónicos por Crianças (3-8 anos) (Públicos e Consumos de Media), Lisboa, Entidade Reguladora para a Comunicação Social [eBook], retirado de: http://www.erc.pt/documentos/Crescendoentreecras/files/downloads/crescendo-en tre-ecras.pdf.

Ponte, Cristina, Teresa Sofia Castro, e Susana Batista (2018), “ ‘Mi hermano lo utiliza. Mis padres lo utilizan. Cualquiera de nosotros coge un Ipad y lo utiliza': la influencia familiar en las actividades digitales de niños portugueses de 6-8 años", em Estefanía Jiménez, Maialen Garmendia e Miguel Ángel Casado (orgs.), Entre Selfies y Whatsapps. Oportunidades y Riesgos para la Infancia y la Adolescencia Conectada (vol. 78), Barcelona, Gedisa Editorial.

Rocha, Cristina, e Manuela Ferreira (2016), “ Porque agora as crianças são bebés!': mudanças percebidas na educação familiar e no jardim de infância por educadoras de infância em Portugal - o caso da alimentação", Trama Interdisciplinar, 7 (2), pp. 125-145. 
Simões, José Alberto, Cristina Ponte, Eduarda Ferreira, Juliana Doretto, e Celiana Azevedo (2014), Crianças e Meios Digitais Móveis em Portugal. Resultados Nacionais do Projeto Net Children Go Mobile. Lisboa, CESNova, FCSH/Nova.

Smahel, David, e Michelle Wright (2014), The Meaning of Online Problematic Situations for Children. Results of Qualitative Cross-Cultural Investigation in Nine European Countries, Londres, LSE, EU Kids Online, ISSN 2045-256X.

Valkenburg, Patti, Marina Krcmar, Allerd Peters, e Nies Marseille (1999), "Developing a scale to assess three different styles of television mediation: 'instrutive mediation', 'restrictive mediation' and 'social coviewing'", Journal of Broadcasting and Eletronic Media, 43 (1), pp. 52-66.

Vittrup, Brigitte, Sharla Snider, Katherine Kensinger Rose, e Jacqueline Rippy (2016), "Parental perceptions of the role of media and technology in their young children's lives", Journal of Early Childhood Research, 14 (1), pp. 43-54.

Zaman, Bieke, Jeroen Vanattenhoven, Evelien de Ferrerre, e Jan van Looy (2016), "A qualitative inquiry into the contextualized parental mediation practices of young children's digital media use at home", Journal of Broadcasting E Electronic Media, 60 (1), pp. 1-22.

Cristina Ponte. Professora associada com agregação no Departamento de Ciências da Comunicação e investigadora integrada no ICNOVA. Faculdade de Ciências Sociais e Humanas, Universidade Nova de Lisboa. E-mail: cristina.ponte@fcsh.unl.pt.

José Alberto Simões. Professor auxiliar no Departamento de Sociologia e investigador Integrado no CICS.NOVA, Faculdade de Ciências Sociais e Humanas, Universidade Nova de Lisboa. E-mail: sjav@fcsh.unl.pt.

Susana Batista. Investigadora integrada CICS.NOVA e professora auxiliar convidada, Faculdade de Ciências Sociais e Humanas, Universidade Nova de Lisboa. E-mail: susanabatista@fcsh.unl.pt

Teresa Sofia Castro. Bolseira de pós-doutoramento FCT e investigadora integrada no ICNOVA. Faculdade de Ciências Sociais e Humanas, Universidade Nova de Lisboa.E-mail: tcastro@fcsh.unl.pt

Receção: 20 de julho de 2017 Aprovação: 16 de janeiro de 2018 